\title{
Supplementary Material \\ Conformational Analysis of Heparin-Analogue Pentasaccharides by Nuclear Magnetic Resonance Spectroscopy and Molecular Dynamics Simulations
}

\author{
Dedicated to the memory of our colleague and dear friend, \\ Dr. István Komáromi.
}

Gábor Balogh, ${ }^{\text {a\# }}$ Tamás Gyöngyösi, ${ }^{\text {b,c\# }}$ István Timári, ${ }^{\text {b\# }}$ Mihály Herczeg, ${ }^{\text {dee }}$ Anikó Borbás, ${ }^{d}$ S. Kashif Sadiq, ${ }^{\mathrm{f}, \mathrm{g}^{*}}$ Krisztina Fehér, ${ }^{\mathrm{c} *}$ Katalin E. Kövérb,c*

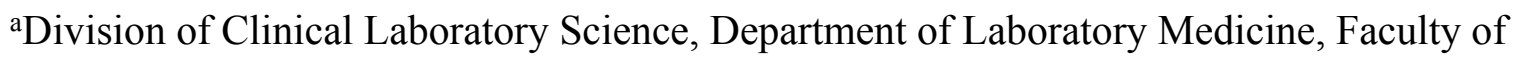
Medicine, University of Debrecen, Nagyerdei krt. 98, H-4032 Debrecen, Hungary

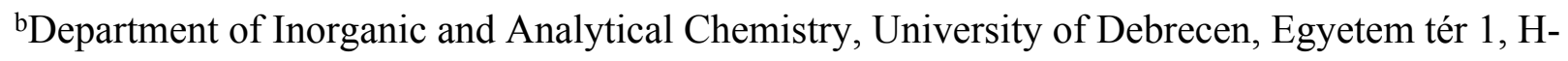
4032 Debrecen, Hungary

'MTA-DE Molecular Recognition and Interaction Research Group, University of Debrecen, Egyetem tér 1, H-4032 Debrecen, Hungary

${ }^{\mathrm{d} D e p a r t m e n t}$ of Pharmaceutical Chemistry, University of Debrecen, Egyetem tér 1, H-4032 Debrecen, Hungary

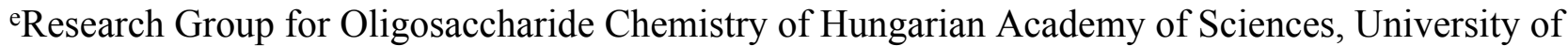
Debrecen, Egyetem tér 1, H-4032 Debrecen, Hungary

fHeidelberg Institute for Theoretical Studies, Schloss-Wolfsbrunnenweg 35, 69118 Heidelberg, Germany

gEuropean Molecular Biology Laboratory, Meyerhofstrasse 1, 69117 Heidelberg, Germany

\#these authors contributed equally

*corresponding authors

E-mail: kover@science.unideb.hu 
E-mail: feher.krisztina@,science.unideb.hu

E-Mail: kashif.sadiq@embl.de

Supplementary Table S1 Experimental homonuclear vicinal coupling constants in the different rings determined for the derivatives $\mathbf{1}, \mathbf{2}$ and $\mathbf{3}$.

\begin{tabular}{|c|c|c|c|c|}
\hline & ${ }^{3} \mathbf{J}_{\mathbf{H}, \mathbf{H}}(\mathrm{Hz})$ & 1 & 2 & 3 \\
\hline \multirow{3}{*}{$\mathrm{D}$ ring } & ${ }^{3} \mathbf{J}_{1,2}$ & 3.9 & 3.8 & 3.6 \\
\hline & ${ }^{3} \mathbf{J}_{2,3}$ & 9.8 & 10.0 & 10.1 \\
\hline & ${ }^{3} \mathbf{J}_{3,4}$ & 9.7 & 9.7 & 9.6 \\
\hline \multirow{3}{*}{ E ring } & ${ }^{3} \mathbf{J}_{1,2}$ & 8.4 & 7.8 & 7.8 \\
\hline & ${ }^{3} \mathbf{J}_{2,3}$ & 9.0 & 9.3 & 8.5 \\
\hline & ${ }^{3} \mathbf{J}_{3,4}$ & 9.4 & 9.4 & 9.4 \\
\hline \multirow{3}{*}{$F$ ring } & ${ }^{3} \mathbf{J}_{1,2}$ & 3.7 & 3.6 & 3.7 \\
\hline & ${ }^{3} \mathbf{J}_{2,3}$ & 10.0 & 9.9 & 10.2 \\
\hline & ${ }^{3} \mathbf{J}_{3,4}$ & 9.8 & 9.7 & 9.6 \\
\hline \multirow{3}{*}{$\mathrm{G}$ ring } & ${ }^{3} \mathbf{J}_{1,2}$ & 2.9 & 3.0 & 3.3 \\
\hline & ${ }^{3} \mathbf{J}_{2,3}$ & 5.7 & 4.9 & 6.0 \\
\hline & ${ }^{3} \mathbf{J}_{3,4}$ & 3.0 & 2.9 & 3.2 \\
\hline \multirow{3}{*}{$\mathrm{H}$ ring } & ${ }^{3} \mathbf{J}_{1,2}$ & 3.7 & 3.7 & 3.7 \\
\hline & ${ }^{3} \mathbf{J}_{2,3}$ & 9.6 & 9.6 & 9.5 \\
\hline & ${ }^{3} \mathbf{J}_{3,4}$ & 9.6 & 9.4 & 9.4 \\
\hline
\end{tabular}


Supplementary Table S2 Comparison of the distances from equilibrium MD corresponding to Nuclear Overhauser Effects (NOE) between ${ }^{1} \mathrm{H}$ nuclei. The standard deviation of the NOE derived distances are ca. 0.1-0.15 ̊.

\begin{tabular}{|c|c|c|c|c|c|c|c|c|}
\hline & \multirow[b]{2}{*}{$\begin{array}{c}{ }^{1} \mathbf{H} \\
\text { nuclei }\end{array}$} & \multirow[b]{2}{*}{$\begin{array}{c}{ }^{1} \mathbf{H} \\
\text { nuclei }\end{array}$} & \multicolumn{2}{|c|}{1} & \multicolumn{2}{|c|}{2} & \multicolumn{2}{|c|}{3} \\
\hline & & & $\begin{array}{c}\text { Distance } \\
\text { estimated } \\
\text { from } \\
\text { NOE (Á) }\end{array}$ & $\begin{array}{c}\text { Average } \\
\text { distance } \pm \\
\text { standard } \\
\text { deviation } \\
\text { from MD } \\
\text { (Å) }\end{array}$ & $\begin{array}{c}\text { Distance } \\
\text { estimated } \\
\text { from } \\
\text { NOE (Á) }\end{array}$ & $\begin{array}{c}\text { Average } \\
\text { distance } \pm \\
\text { standard } \\
\text { deviation } \\
\text { from MD } \\
\text { (Á) }\end{array}$ & $\begin{array}{c}\text { Distance } \\
\text { estimated } \\
\text { from } \\
\text { NOE (Á) }\end{array}$ & $\begin{array}{c}\text { Average } \\
\text { distance } \pm \\
\text { standard } \\
\text { deviation } \\
\text { from MD } \\
\text { (Á) }\end{array}$ \\
\hline 1 & D1 & E4 & 2.5 & $2.9 \pm 0.3$ & 2.5 & $2.9 \pm 0.3$ & 2.6 & $2.9 \pm 0.3$ \\
\hline 2 & D1 & E5 & 3.8 & $4.3 \pm 0.3$ & 3.4 & $4.3 \pm 0.3$ & 3.0 & $4.3 \pm 0.3$ \\
\hline 3 & D1 & D3 & 3.3 & $3.8 \pm 0.1$ & 2.7 & $3.8 \pm 0.1$ & 2.5 & $3.8 \pm 0.1$ \\
\hline 4 & D1 & E3 & 3.3 & $2.9 \pm 0.4$ & 2.7 & $2.9 \pm 0.4$ & 2.5 & $2.9 \pm 0.4$ \\
\hline 5 & D6 & D5 & 2.6 & $2.5 \pm 0.3$ & 2.7 & $2.6 \pm 0.3$ & 2.8 & $2.8 \pm 0.3$ \\
\hline 6 & E1 & F6 & 2.7 & $2.6 \pm 0.4$ & 2.9 & $2.6 \pm 0.4$ & 2.8 & $2.7 \pm 0.5$ \\
\hline 7 & E1 & F4 & 2.6 & $2.5 \pm 0.2$ & 2.7 & $2.4 \pm 0.2$ & 2.2 & $2.4 \pm 0.2$ \\
\hline 8 & E1 & E4 & 3.2 & $4.0 \pm 0.1$ & 3.3 & $4.0 \pm 0.1$ & 3.2 & $4.0 \pm 0.1$ \\
\hline 9 & E1 & E3 & 2.8 & $2.8 \pm 0.2$ & 2.8 & $2.8 \pm 0.2$ & 2.6 & $2.8 \pm 0.2$ \\
\hline 10 & $\mathrm{~F} 1$ & F3 & 3.6 & $3.8 \pm 0.1$ & 3.4 & $3.8 \pm 0.1$ & 3.5 & $3.8 \pm 0.1$ \\
\hline 11 & F1 & F2 & 2.5 & $2.4 \pm 0.1$ & 2.5 & $2.4 \pm 0.1$ & 2.5 & $2.4 \pm 0.1$ \\
\hline 12 & $\mathrm{~F} 1$ & G4 & 2.6 & $2.7 \pm 0.3$ & 2.8 & $2.8 \pm 0.3$ & 2.7 & $2.8 \pm 0.3$ \\
\hline 13 & $\mathrm{~F} 1$ & G3 & 2.4 & $2.4 \pm 0.3$ & 2.4 & $2.3 \pm 0.2$ & 2.3 & $2.3 \pm 0.2$ \\
\hline 14 & G1 & $\mathrm{H} 3$ & 3.6 & $4.3 \pm 0.6$ & 3.5 & $4.5 \pm 0.2$ & 3.6 & $4.4 \pm 0.5$ \\
\hline 15 & G1 & H6 & 3.3 & $2.9 \pm 0.7$ & 2.7 & $2.8 \pm 0.5$ & 2.7 & $2.8 \pm 0.6$ \\
\hline 16 & G1 & H6' & 2.9 & $3.1 \pm 0.9$ & 3.2 & $2.5 \pm 0.6$ & 3.2 & $2.6 \pm 0.7$ \\
\hline 17 & G1 & H5 & 3.0 & $3.7 \pm 0.4$ & 3.1 & $3.8 \pm 0.3$ & 3.3 & $3.8 \pm 0.4$ \\
\hline 18 & G1 & $\mathrm{H} 4$ & 2.6 & $2.6 \pm 0.4$ & 2.5 & $2.5 \pm 0.3$ & 2.4 & $2.6 \pm 0.3$ \\
\hline 19 & G1 & G3 & 3.3 & $3.2 \pm 0.6$ & 3.2 & $3.0 \pm 0.5$ & 2.4 & $3.1 \pm 0.5$ \\
\hline 20 & G1 & $\mathrm{G} 2$ & 2.9 & $2.9 \pm 0.2$ & 3.1 & $3.0 \pm 0.2$ & 2.8 & $3.0 \pm 0.2$ \\
\hline
\end{tabular}




\begin{tabular}{|c|c|c|c|c|c|c|c|c|}
\hline 21 & G2 & G3 & 2.9 & $2.9 \pm 0.2$ & 2.7 & $3.0 \pm 0.2$ & 2.5 & $2.9 \pm 0.2$ \\
\hline 22 & G4 & G3 & 2.8 & $2.8 \pm 0.2$ & 3.1 & $2.9 \pm 0.2$ & 3.0 & $2.9 \pm 0.2$ \\
\hline 23 & G5 & G4 & 2.4 & $2.4 \pm 0.1$ & 2.5 & $2.4 \pm 0.1$ & 2.4 & $2.4 \pm 0.1$ \\
\hline 24 & G5 & H4 & 4.2 & $3.9 \pm 0.4$ & 4.1 & $4.0 \pm 0.4$ & 4.1 & $4.0 \pm 0.4$ \\
\hline 25 & G5 & G2 & 2.9 & $2.7 \pm 0.7$ & 2.8 & $2.5 \pm 0.5$ & 2.7 & $2.5 \pm 0.6$ \\
\hline
\end{tabular}


Supplementary Figure S1 Time evolution of the Cremer-Pople $\theta$ and $\varphi$ parameters and that of interglycosidic $\varphi$ (in red) and $\psi$ (in blue) torsion angles during the 10 replica equilibrium MD simulations for the different subunits for derivatives $\mathbf{1}$ in a), for $\mathbf{2}$ in b) and for $\mathbf{3}$ in c).

a)

D

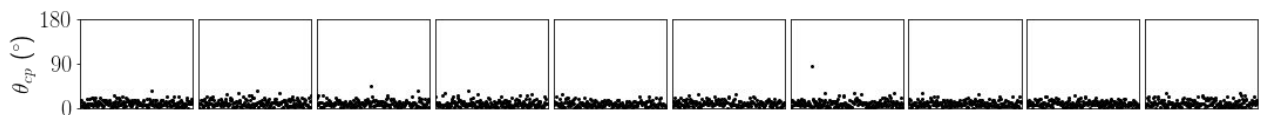

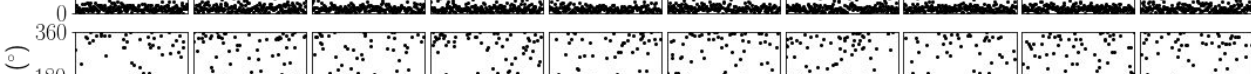

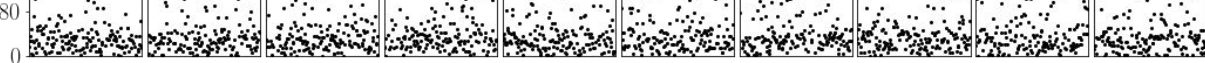

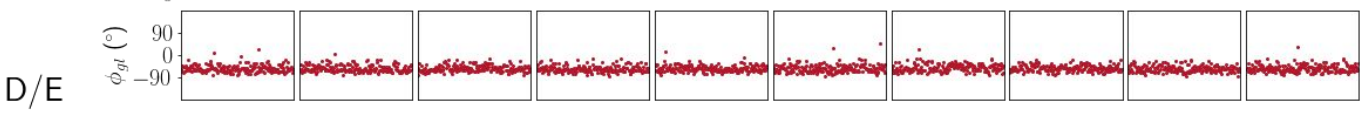

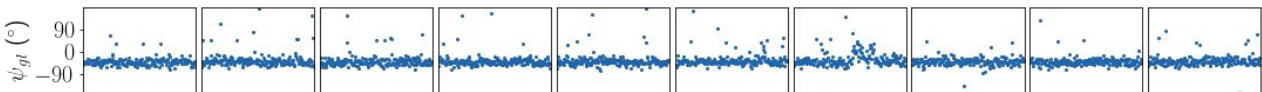

E

$\sigma_{0}^{180} 90-10$

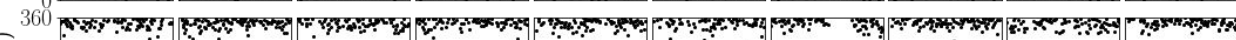
○. 180

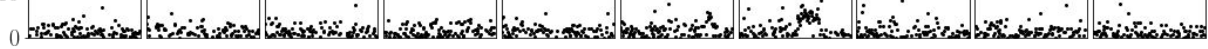

$\mathrm{E} / \mathrm{F}$

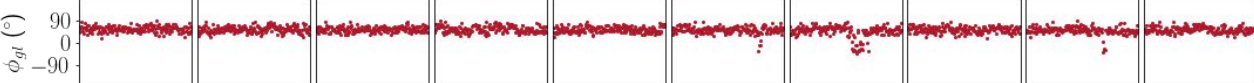
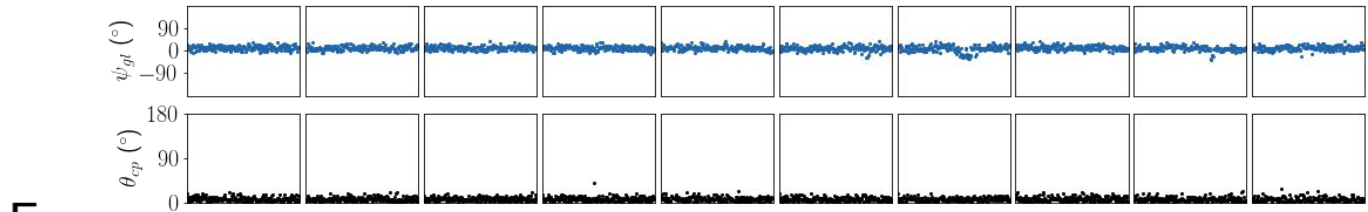

F

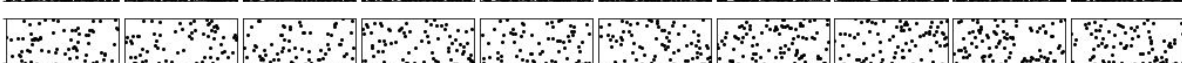

o 180 of

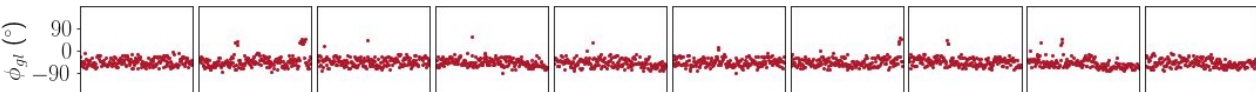

$F / G$
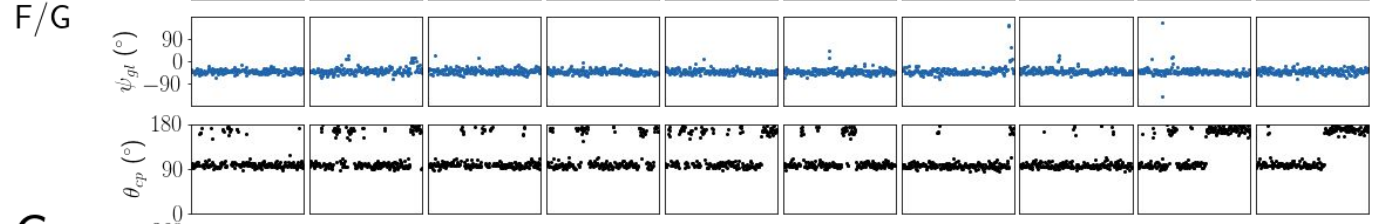

$G$
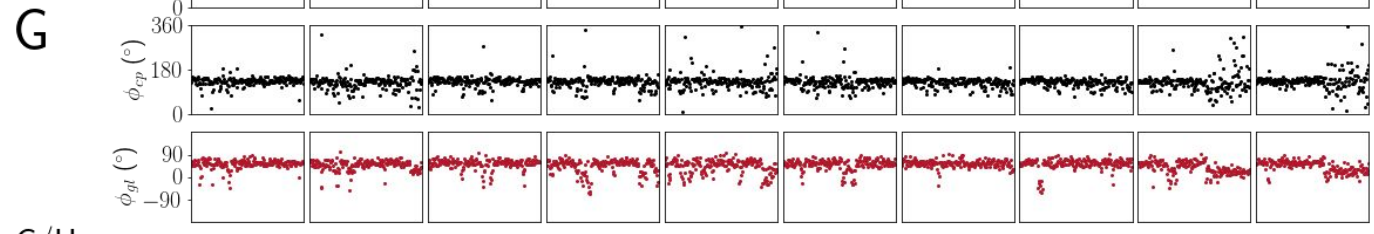

G/H
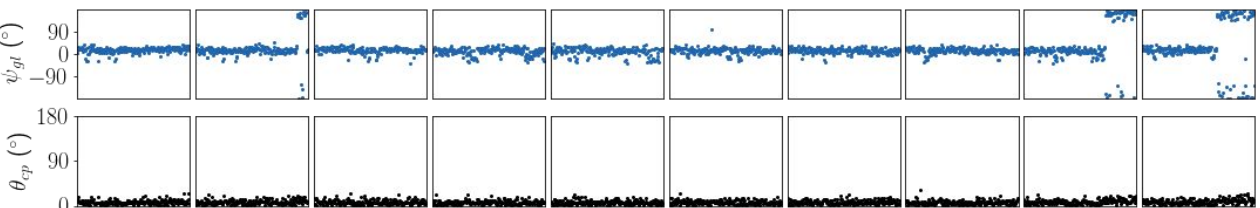

$H$

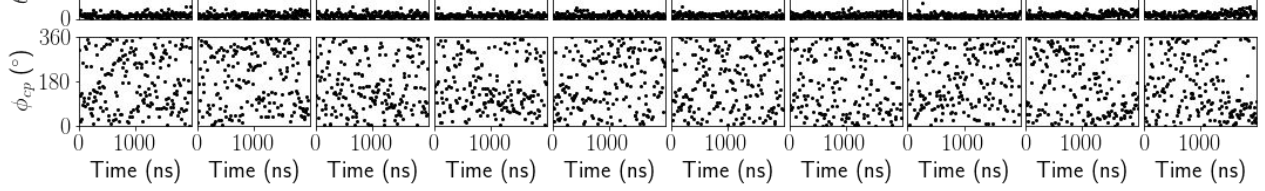


b)

D

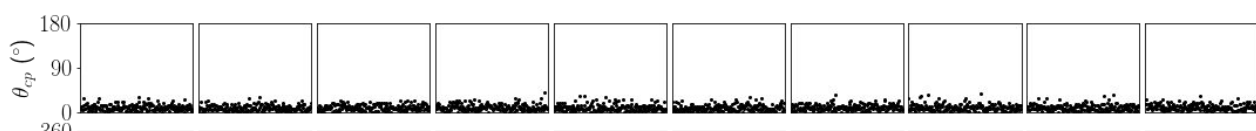

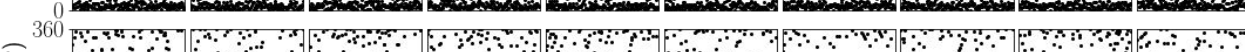

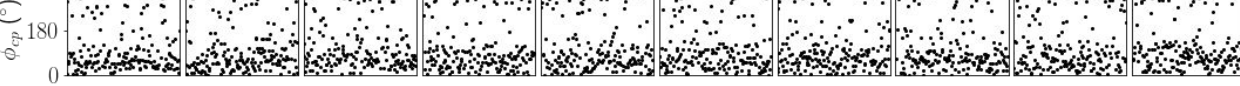

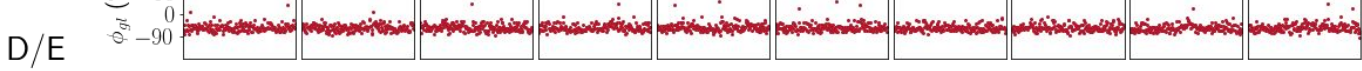

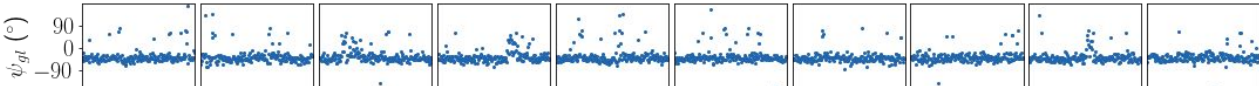

$E$

$\sigma_{0}^{180} 90-10$

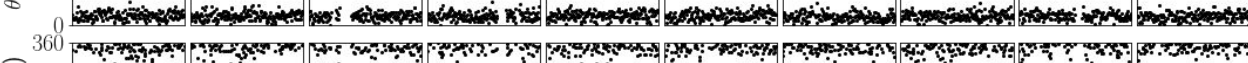
of 180

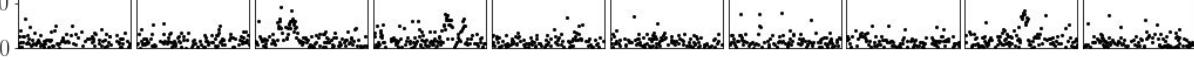

$E / F$

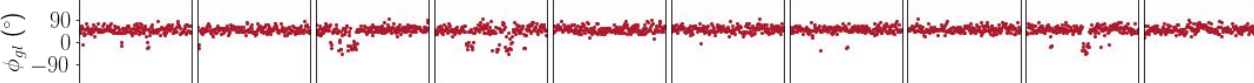

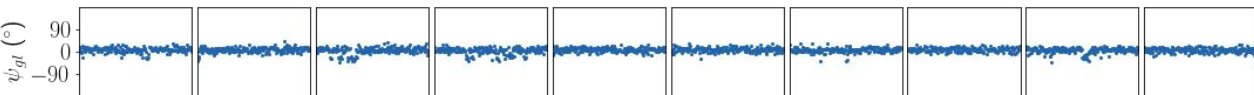

F
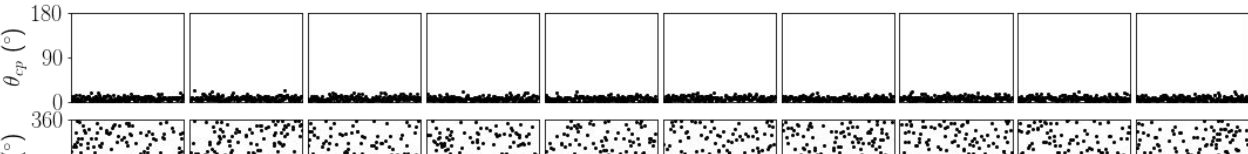

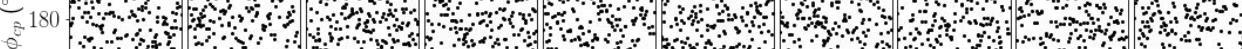

$0 \%$ on

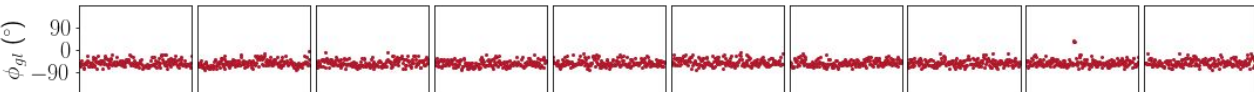

$\mathrm{F} / \mathrm{G}$
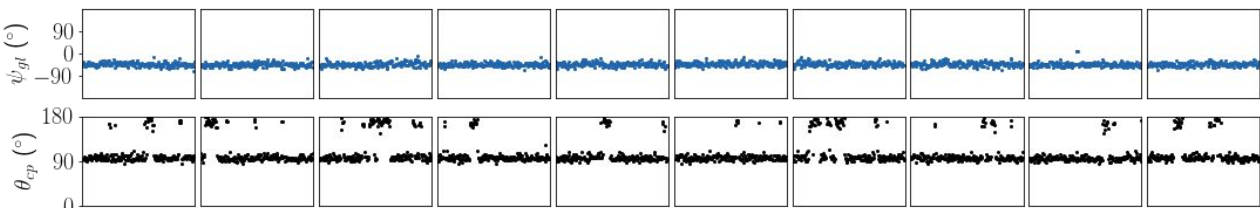

G

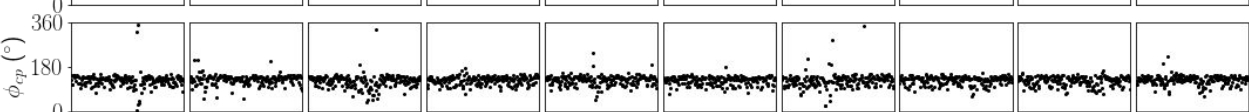

$\mathrm{G} / \mathrm{H}$

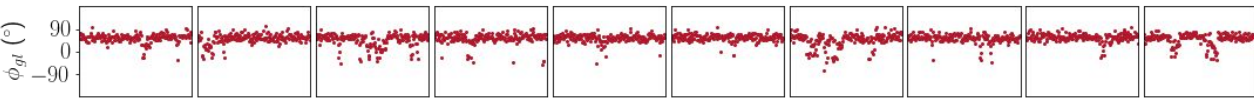

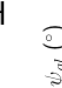
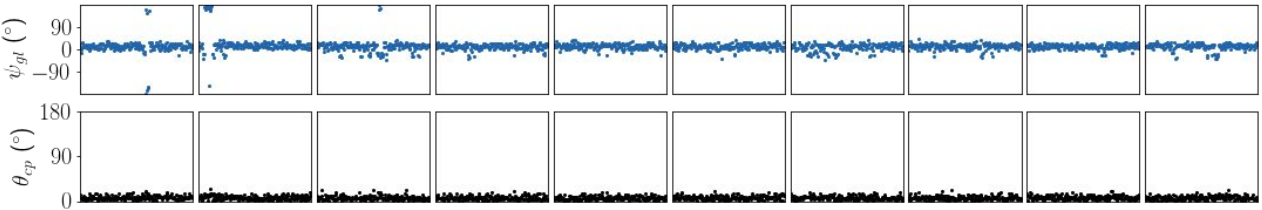

$\mathrm{H} \quad{ }^{360} \mathrm{p}$

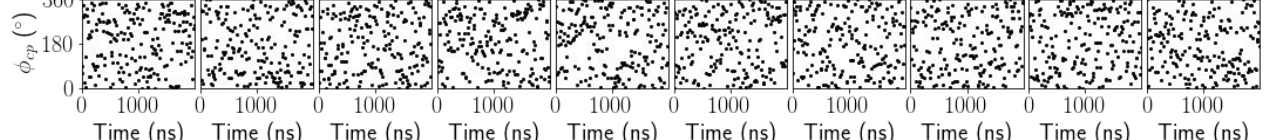

c) 


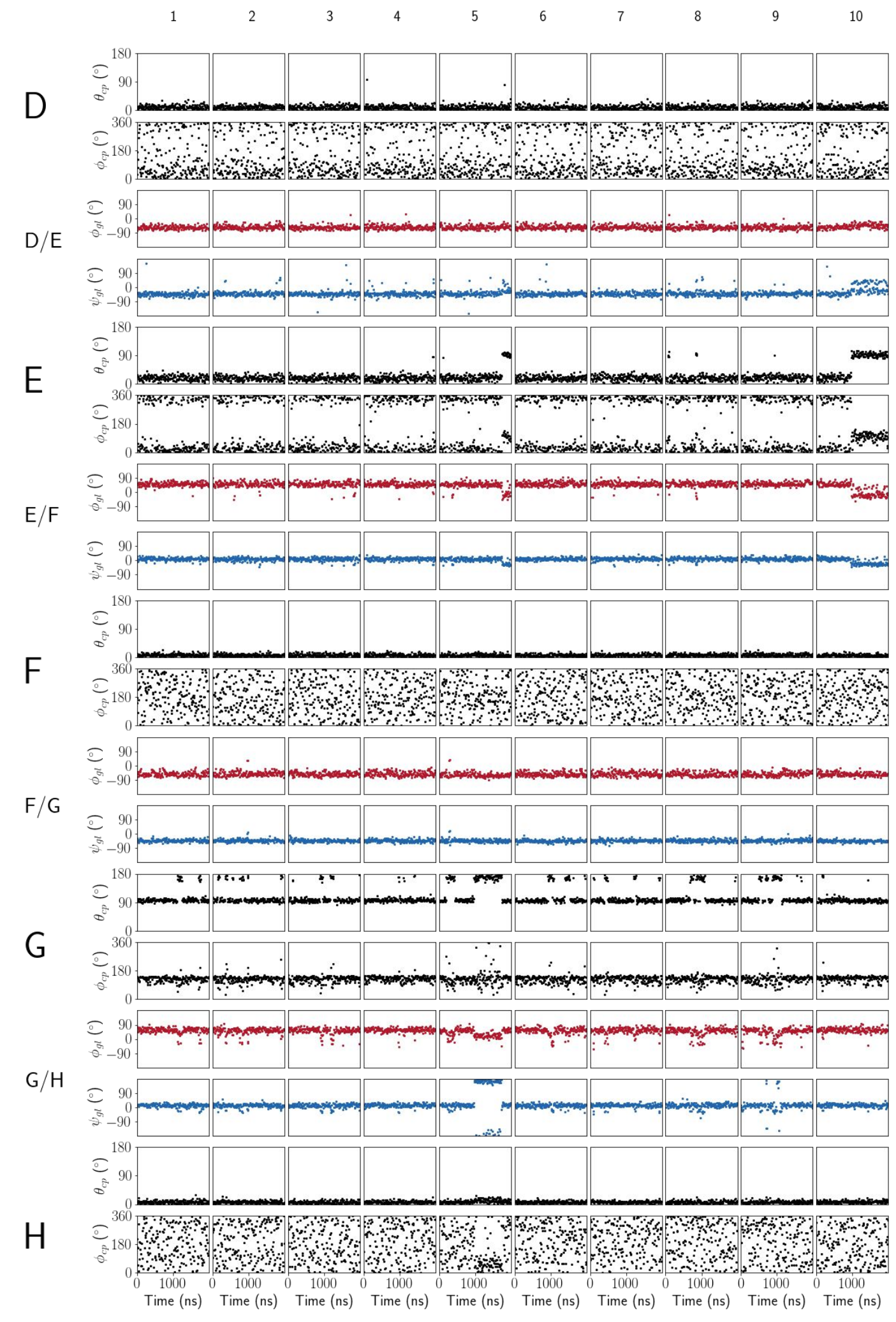


Supplementary Figure S2 Calculated RMSD values of the conformations in the trajectories using bound pentasaccharide in 1E03 as a reference.

1
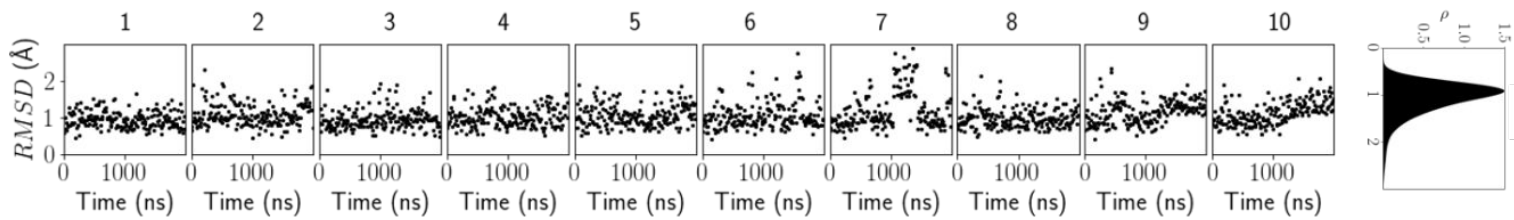

2
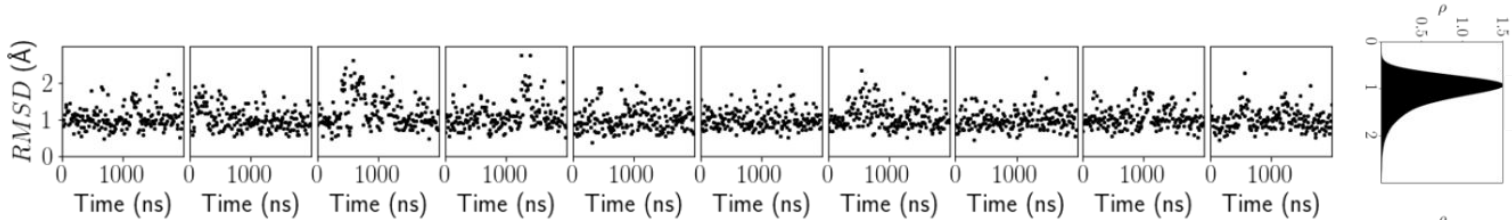

3
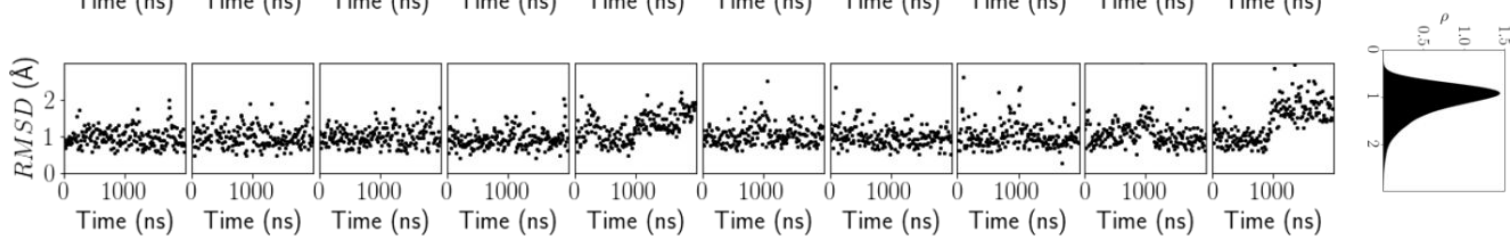